\title{
PREVALENCE OF PERIPHERAL NEUROPATHY SECONDARY TO CHEMOTHERAPY IN WOMEN WITH BREAST CANCER
}

Victor Domingos Lisita Rosa', Amanda Laíza dos Reis Mota', Diego Vinícius Gonçalves Santana', Lanuscia Morais de Santana1, Beatriz Rocha Almeida'1, Hugo Francisco da Fonseca Neto'1, Lucas Pierson Brom Vieira', Ruffo de Freitas-Junior $^{1}$

${ }^{1}$ Hospital das Clínicas, Universidade Federal de Goiás - Goiânia (GO), Brazil.

Objective: This study aims to assess the prevalence of peripheral neuropathy in patients undergoing potentially neurotoxic chemotherapy for breast cancer. Methodology: This is a longitudinal study to assess the prevalence of peripheral neuropathy in women with breast cancer undergoing chemotherapy at the Hospital das Clínicas, Universidade Federal de Goiás (HC-UFG). The study was approved by the Ethics and Research Committee of HC-UFG, and all participants signed an informed consent form. For the data collection, two instruments were used, namely, Sociodemographic Form and Antineoplastic-Induced Neurotoxicity Questionnaire (AINQ). The Microsoft ${ }^{\circledR}$ Excel 2007 was used to tabulate the data, and the statistical analysis was performed using the SPSS ${ }^{\circledR}$ for Windows ${ }^{\circledR}$ program, version 22.0. Results: A total of 30 patients were included in this study. The average age was 51.2 years, and the sociodemographic evaluation showed that the majority of the participants were married (60\%), $46.7 \%$ had one or two children, $23.3 \%$ were unemployed, and the schooling time was 9.88 years. As for the analysis of the AINQ in the three moments of the study, there was no statistical difference between the use of chemotherapy, body mass index, and age for a higher incidence of symptoms of peripheral neuropathy. The prevalence of symptoms of peripheral neuropathy in the sample was $83.3 \%$, with orofacial symptoms being the most reported (grades 1 and 2). Conclusion: The study confirmed the high prevalence of neurotoxicity symptoms related to chemotherapy, both acute and chronic. The persistence of chronic symptoms suggests that, if chemotherapy-induced peripheral neuropathy (CIPN) is correctly diagnosed, it will be possible to control it early, avoiding further damage to patients.

Keywords: Breast Cancer; Neuropathy; Chemotherapy. 